\title{
Negative visuospatial priming in isolation-reared rats: Evidence of resistance to the disruptive effects of amphetamine
}

\author{
Nurith Amitai $^{1,3} \cdot$ Susan Powell ${ }^{1,2} \cdot$ Martin Weber ${ }^{1,4} \cdot$ Neal R. Swerdlow $^{1}$. \\ Jared W. Young ${ }^{1,2}$
}

Published online: 29 July 2015

(C) Psychonomic Society, Inc. 2015

\begin{abstract}
Negative visuospatial priming (NP) represents a quantifiable measure of inhibitory information processing that is disrupted in several neurodevelopmental and psychiatric disorders, including schizophrenia. We developed a novel rodent NP task to investigate mechanisms underlying NP and its role in various disorders, and to test potential therapeutics. In the present studies, we further characterized this novel paradigm by investigating whether NP is disrupted in rats reared in isolation, a developmental manipulation that produces a range of abnormalities in behavior, neurochemistry, and brain structure that mirror aspects of schizophrenia pathology. We also further explored the role of monoaminergic signaling in NP and the effects of isolation rearing by challenging both socially reared and isolation-reared rats with D-amphetamine during the NP task. Although fewer isolation-reared animals learned the complex NP task, those that learned exhibited unaffected NP compared with socially reared rats. Consistent with previous reports, D-amphetamine impaired NP and increased motor impulsivity in socially reared rats. In contrast, Damphetamine did not affect NP or motor impulsivity in isolation-reared rats. These data confirm a monoaminergic
\end{abstract}

Jared W. Young

jaredyoung@ucsd.edu

1 Department of Psychiatry, University of California San Diego, 9500 Gilman Drive MC 0804, La Jolla, CA 92093-0804, USA

2 Research Service, VA San Diego Healthcare System, San Diego, CA, USA

3 Present address: Explora BioLabs, 3030 Bunker Hill Street, Suite 300, San Diego, CA 92109, USA

4 Present address: Neurodegeneration Labs, Department of Neuroscience, Genentech Inc., 1 DNA Way, South San Francisco, CA 94080, USA influence on NP behavior and indicate that rats reared in isolation have altered dopaminergic sensitivity.

Keywords Isolation rearing $\cdot$ Visuospatial priming $\cdot$ Negative priming $\cdot$ Amphetamine $\cdot$ Schizophrenia $\cdot$ Information processing $\cdot$ Rats

\begin{tabular}{ll}
\multicolumn{2}{l}{ Abbreviations } \\
5-HT & serotonin \\
AMPH & D-amphetamine \\
DA & Dopamine \\
ISI & Interstimulus interval \\
ITI & Intertrial interval \\
NP & Negative priming \\
PFC & Prefrontal cortex
\end{tabular}

\section{Introduction}

Many neurodevelopmental and psychiatric disorders are characterized by dysfunctional information processing. Specifically, impairments in inhibitory information processing - defined as the ability to suppress reactions to irrelevant or inappropriate information - may contribute significantly to many of the cognitive and behavioral symptoms of these disorders. Negative priming (NP) constitutes a cognitive-behavioral phenomenon that allows operational assessment of inhibitory information processing in the laboratory.

The negative visuospatial priming (NP) task requires the subject to respond to a target stimulus while simultaneously ignoring a non-target distractor stimulus in pairs of trials. If, during the second trial, the target is presented in a location that previously held the distractor stimulus (see Fig. 1), subjects exhibit lower accuracy and/or slower response times. This 
PRIME TRIAL
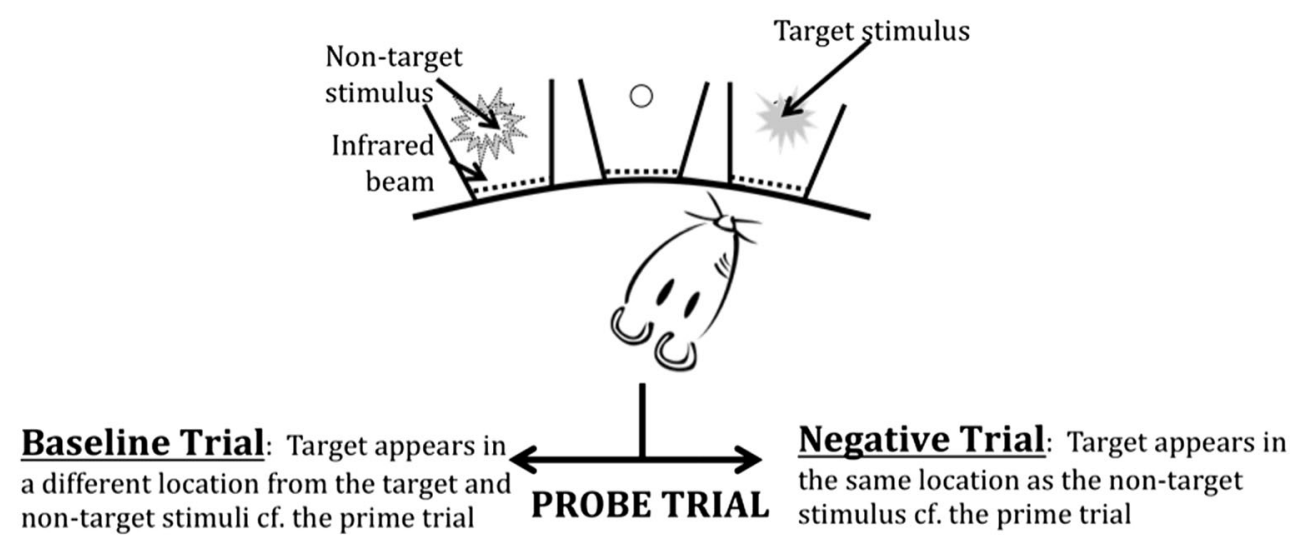

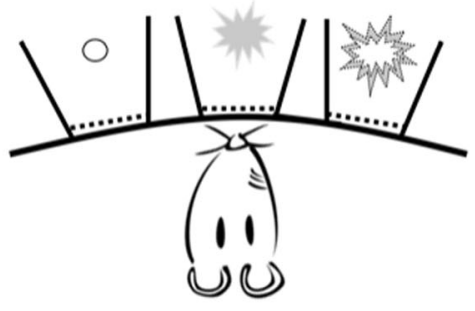

Fig. 1 Schematic representation of the different trial types of negative priming. Prime Trial: The rodent faces a curved wall with three equidistant apertures. Each aperture contains an infrared beam at the entrance to detect nose-poke responses and a LED stimulus light at the rear to present stimuli. During the Prime Trial, the target stimulus (continuous light) and the non-target stimulus (5-Hz flashing light) are briefly presented simultaneously in pseudorandom locations. Probe

decrease in task performance is termed "negative priming" and reflects inhibitory information processing (Tipper, 1985, 2001). NP is disrupted in several neurodevelopmental and psychiatric disorders, including Tourette syndrome and attention deficit hyperactivity disorder, consistent with the impaired inhibitory information processing in these disorders.

While the NP task is well-established in humans, no corresponding animal paradigm measuring negative visuospatial priming had been reported previously. Such paradigms are necessary to facilitate the investigation of mechanisms underlying this measure of inhibitory information processing, and may aid the development of novel therapeutics for disorders characterized by dysfunctional information processing. We recently developed and validated a rodent NP task (Amitai et al., 2013).

Dopamine (DA) signaling has been implicated in the mediation of visuospatial priming (Swerdlow, Hartman, \& Auerbach, 1997; Wylie \& Stout, 2002; Yamaguchi \& Kobayashi, 1998). In our previous study, we therefore examined the effect of the indirect DA agonist, D-amphetamine (AMPH), on NP in our task. We demonstrated that AMPH had a rate-dependent effect on NP: in animals that had high levels of NP in the drug-free state ("high-priming animals"), a low dose $(0.25 \mathrm{mg} / \mathrm{kg})$ of AMPH reduced NP. In contrast, in animals with low levels of NP in the drug-free state ("low-

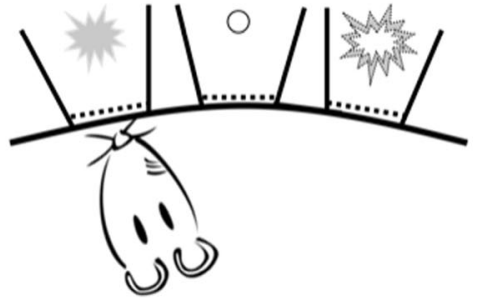

Trial: After a short period of time (interstimulus interval, ISI), a Probe Trial follows. In the case of a Baseline Trial, the location of the target stimulus in the Probe Trial is unrelated to the location of either stimulus in the Prime Trial. In the case of a Negative Trial, the target stimulus in the Probe Trial is located in the same aperture that contained the non-target stimulus during the preceding Prime Trial

priming animals"), the same dose of AMPH increased NP. At higher doses, AMPH abolished NP in all animals (Amitai et al., 2013). Our findings suggested the possibility of an "optimal" level of DA signaling that is required for maximal NP.

In the present studies, we strove to further characterize our NP model by investigating how NP in our task was affected by isolation rearing of rats, a widely studied animal model relevant to neurodevelopmental and psychiatric disorders, including schizophrenia. Many of the cognitive and behavioral symptoms of schizophrenia may reflect underlying pervasive information processing deficits (Braff, 1993; Nuechterlein \& Dawson, 1984). NP is disrupted in schizophrenia (Beech, Powell, McWilliam, \& Claridge, 1989; Elkins \& Cromwell, 1994). Intriguingly, these NP deficits are less pronounced in patients treated with the antipsychotic clozapine (MacQueen, Galway, Goldberg, \& Tipper, 2003). Clozapine also ameliorates numerous other symptoms of schizophrenia, including affective symptoms (Meltzer, 2002) and cognitive deficits (Buchanan, Holstein, \& Breier, 1994; Meltzer \& McGurk, 1999; Potkin, Fleming, Jin, \& Gulasekaram, 2001) that do not respond to traditional antipsychotics (Harvey, Rabinowitz, Eerdekens, \& Davidson, 2005; Jibson \& Tandon, 1998; Mortimer, 1997). These findings support the possibility that improving inhibitory processing in patients may alleviate some psychiatric symptoms. A better 
understanding of the inhibitory processing deficits in disorders like schizophrenia is therefore likely to yield valuable insights into the neural circuits involved in psychiatric pathology, and possibly identify targets for therapeutic intervention.

Socially isolating rodents at weaning profoundly impacts their behavioral and neurobiological development during a period when they acquire important behaviors such as play behavior (Einon \& Morgan, 1977). This early-life disruption models childhood stressors that increase vulnerability for neuropsychiatric disorders, including schizophrenia (Agid et al., 1999; Lim, Chong, \& Keefe, 2009). Despite being minimally invasive (no pharmacological or surgical interventions are used), isolation rearing produces permanent alterations in brain structure, neurotransmission, behavior, and cognition that reproduce some abnormalities observed in schizophrenia (Fone \& Porkess, 2008; Lapiz et al., 2003; Powell, 2010). Notably, these effects are developmentally specific: they result only when animals are isolated as young juveniles post weaning and not when isolated as adults (Cilia, Reavill, Hagan, \& Jones, 2001; Wilkinson et al., 1994). Moreover, many of these abnormalities do not manifest prior to puberty (Bakshi \& Geyer, 1999). This time course is consistent with the development of symptoms in schizophrenia (Weinberger, 1987). For all of these reasons, isolation rearing is an attractive model for neurobiological, cognitive, and behavioral aspects of neuropsychiatric disorders (including schizophrenia) that avoids many of the confounds arising from pharmacological or surgical manipulations.

Isolation rearing induces deficits of information processing such as impaired prepulse inhibition (Cilia, Hatcher, Reavill, \& Jones, 2005; Cilia et al., 2001; Geyer, Wilkinson, Humby, \& Robbins, 1993; Varty \& Geyer, 1998; Varty \& Higgins, 1995; Wilkinson et al., 1994) that can be attenuated by antipsychotics (Bakshi, Swerdlow, Braff, \& Geyer, 1998; Cilia et al., 2001; Geyer et al., 1993; Varty \& Higgins, 1995; Wilkinson et al., 1994), just as PPI deficits in schizophrenia patients are diminished by antipsychotics (Braff et al., 1978; Braff, Geyer, \& Swerdlow, 2001; Kumari \& Gray, 1999). To date, however, the effects of isolation rearing on information processing as measured by NP have not been investigated.

In light of the role of DA signaling in NP, it is important to note that isolation rearing induces abnormalities of dopaminergic function (Blanc et al., 1980; Fulford \& Marsden, 1998; Hall, 1998; Heidbreder et al., 2000; Jones, Hernandez, Kendall, Marsden, \& Robbins, 1992; Robbins, Jones, \& Wilkinson, 1996). Dysfunctional DA signaling is also implicated in hypotheses about the pathophysiology of schizophrenia (Davis, Kahn, Ko, \& Davidson, 1991). Both schizophrenia patients (Abi-Dargham et al., 1998; Breier, Malhotra, Pinals, Weisenfeld, \& Pickar, 1997; Laruelle et al., 1996) and isolation-reared rodents (Jones et al., 1992; Jones, Marsden, \& Robbins, 1990; Sahakian, Robbins, Morgan, \& Iversen, 1975 ) exhibit increases in behavioral effects and DA release in response to indirect DA agonists. We therefore investigated the effect of AMPH on NP performance in isolates and their socially reared littermates.

We hypothesized that isolation-reared rats would exhibit NP deficits, mirroring the NP deficits found in schizophrenia patients. Consequently, given our previous finding that low doses of AMPH improve NP in animals with low NP in the drug-free state, we hypothesized that AMPH would normalize these low levels of NP in isolation-reared rats.

\section{Materials and methods}

\section{Animals}

Fifty-five male Long-Evans rats (Charles River Laboratories, Wilmington, MA, USA) were weaned at 24 days postnatal. Twenty-nine of the pups were single-housed (isolation-reared rats or isolates), while the 26 remaining pups were housed in groups of either three (24 rats) or two (two rats) (socially reared rats or socials). These rats were tested in startle and between-session probabilistic learning paradigms (Amitai et al., 2014) prior to training in the NP task at week 20 postweaning. Rats were allowed to reach a body weight of at least $300 \mathrm{~g}$ before initiation of food restriction, which was calibrated to keep rats at $90 \%$ of their free-feeding weight. Water was available ad libitum at all times except during testing. Rats were housed on a $12 \mathrm{~h}: 12 \mathrm{~h}$ reversed light-dark cycle (lights off at 7:00 am); all behavioral testing was conducted during the animals' dark cycle. Animals were treated in accordance with the guidelines of the American Association for the Accreditation of Laboratory Animal Care and the National Research Council's Guide for Care and Use of Laboratory Animals. All experiments were approved by the Animal Care and Use Committee of the University of California San Diego.

\section{Drugs}

D-Amphetamine sulfate (Sigma, St. Louis, MO, USA) was dissolved in $0.9 \%$ saline solution and administered by subcutaneous injection in a volume of $1 \mathrm{ml} / \mathrm{kg}$ at a dose of $0.25 \mathrm{mg} / \mathrm{kg}$ (free base). This dose was chosen because it produced baseline-dependent effects on NP values in the rat NP task, while not disrupting successful performance of the task itself which occurred at higher doses (Amitai et al., 2013).

\section{Apparatus}

Training and testing were conducted in nine-hole operant testing chambers enclosed in ventilated sound-attenuating chambers (Med Associates Inc., St. Albans, VT, USA and Lafayette Instrument Company, Lafayette, IN, USA). Each testing 
chamber contained a curved rear wall with nine contiguous apertures. Metal inserts covered six of the apertures, leaving open apertures 3, 5, and 7. An infrared beam located at the entrance of each aperture detected nose-poke responses, and a stimulus light was located at the rear of each aperture. Liquid reinforcement in the form of strawberry milkshake (Nesquik ${ }^{\circledR}$ plus non-fat milk, $40 \mu \mathrm{l}$ ) could be delivered into a magazine located in the opposite wall via peristaltic pump; an infrared beam detected head entries into the magazine. A house light was located in the middle of the chamber ceiling. The control of stimuli and recording of responses were managed by a SmartCtrl Package 8-In/16-Out with additional interfacing by MED-PC for Windows (Med Associates Inc., St. Albans, VT, USA) using custom programming.

\section{Behavioral procedure}

Rats were trained in the NP task as described previously (Amitai et al., 2013). To learn to retrieve the liquid reward from the magazine, rats were exposed to the test chamber on two consecutive days in 10-min sessions, during which a $40-\mu l$ increment of strawberry milkshake was delivered noncontingently into the magazine every $15 \mathrm{~s}$ (Habituation 1). Delivery of the reward was accompanied by illumination of the magazine light. Head entries into the magazine to consume the reward led to extinction of the magazine light until the delivery of the next reward.

Next, the rats were trained to nose poke for the reward during daily 30-min sessions, in which all three apertures were illuminated until a nose-poke response occurred (Habituation 2). A nose poke into any aperture resulted in extinction of all aperture lights and delivery of a reward into the food magazine. Head entries into the magazine initiated a 4-s intertrial interval (ITI), after which all apertures were again illuminated. To prevent the establishment of positional biases, a streak of five consecutive responses into the same aperture resulted in this aperture no longer being illuminated or responsive until the rat nose poked at least once into each of the two remaining apertures.

Once rats had performed $>60$ responses for two consecutive sessions, they were trained to distinguish between target and non-target visual stimuli (Visual Discrimination). All trials were initiated by a head entry into the food magazine. An initial noncontingent liquid reward was delivered into the magazine at the start of each session to facilitate initiation of the first trial. After a 4-s ITI, the target stimulus (a continuous light) and the non-target distractor stimulus (a 5-Hz flashing light) were presented simultaneously in two of the response apertures. The location of the target and non-target stimuli varied pseudorandomly between trials, so that each location contained the stimuli an equal number of times per session, but their location was not predictable on any given trial. A nose poke in the aperture containing the target stimulus that occurred during stimulus presentation or within a 1-s limited hold period after the end of the stimulus (correct response) resulted in the delivery of a reward into the food magazine, along with illumination of the magazine light. Nose pokes into the aperture containing the non-target stimulus (false alarm) or into an unlit aperture (incorrect response) were punished by a 4-s timeout, marked by illumination of the house light and no delivery of the reward. Nose pokes in any aperture made before presentation of the target and non-target stimuli (premature responses) likewise resulted in a timeout and no reward. At the end of the timeout, the house light was extinguished and the magazine light illuminated to prompt the animal to initiate the next trial via a head entry into the magazine. The magazine light was extinguished after removal of the head from the magazine. Each session lasted $30 \mathrm{~min}$ or until the animal had completed 120 trials, whichever occurred first. During the initial sessions, the target and non-target stimuli were continuously illuminated until a nose poke occurred. Once rats had performed at least 60 correct responses, the target and non-target stimulus durations were limited to $10 \mathrm{~s}$. No response within the stimulus presentation resulted in recording of an omission error and punishment of rats with a timeout and no reward. Rats were trained until they performed at least 60 correct responses per session and selected the target stimulus with $>60 \%$ accuracy. On average, rats required around 15 sessions to reach criterion performance.

Rats were then trained on the negative priming (NP) task. See Fig. 1 for schematic representation of task and Fig. 2 for a flowchart of task performance. Again, all trials were initiated by a head entry into the magazine. After a 4-s ITI, the animal was presented with the prime, consisting of $0.5 \mathrm{~s}$ of simultaneous presentation of the target and non-target stimuli. Nosepoke responses to the prime in any location were recorded, but had no consequence. The stimuli were then extinguished, and a 0.3-s interstimulus interval (ISI) followed. After the ISI, the animal was presented with the probe, consisting of the target and non-target stimuli presented simultaneously in different locations. In half of the trials, the target stimulus during the probe was located in the aperture that had contained no visual stimulus during the prime (baseline trials). In the remaining trials, the target stimulus during the probe was located in the aperture that previously contained the non-target stimulus during the prime (negative trials). Baseline and negative trials alternated pseudorandomly, so that that each trial type was presented equally often during each session, but the trial type was not predictable on any given trial. Responses during the ITI before the onset of the prime stimuli (premature responses) were punished with a timeout period and no reward; responses during the ISI (i.e., between the prime and probe stimuli presentations; ISI responses) were recorded, but had no consequence. Each session lasted 30 min or until the animal had completed 120 trials, whichever occurred first. The duration of the target and non-target stimuli during the 


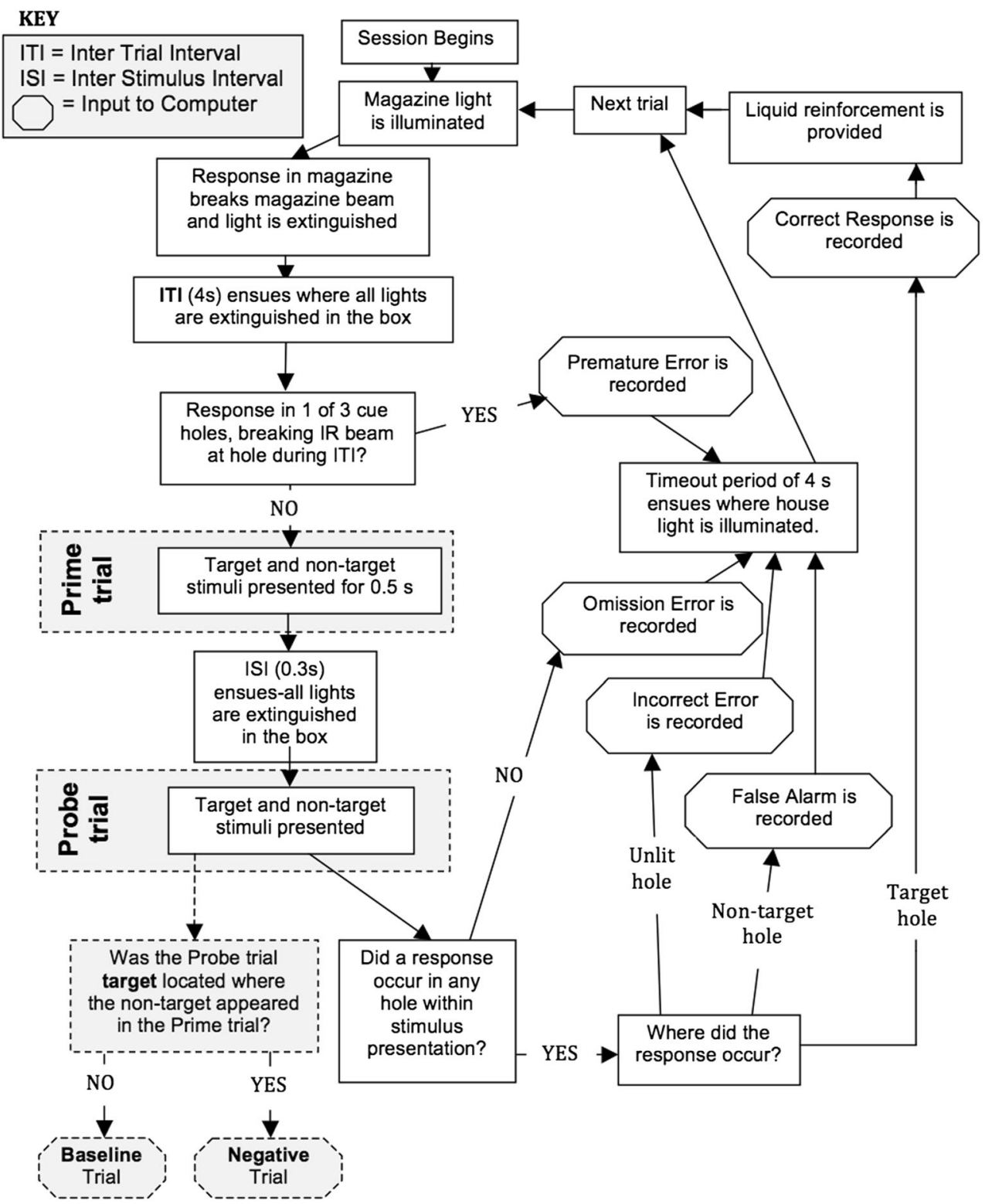

Fig. 2 Flowchart showing the negative visuospatial priming task (VSP). The chart depicts a step-by-step overview of how each trial occurs and how each measure is gathered from the behavior of the rat performing the task. Reprinted from Neuroscience and Biobehavioral Reviews 37(6),
Amitai N, Weber M, Swerdlow NR, Sharp RF, Breier MR, Halberstadt AL, Young JW, A novel visuospatial priming task for rats with relevance to Tourette syndrome and modulation of dopamine levels, pages 11391149, Copyright 2013, with permission from Elsevier

The following measures were calculated to assess task performance:

- Accuracy: Proportion of total responses to stimuli that were correct. Accuracy was only computed if correct responses + incorrect responses + false alarms totaled 10 or more.

$$
\text { Accuracy }=\frac{\text { Correct }}{\text { Correct }+ \text { Incorrect }+ \text { False alarms }}
$$

- Accuracy priming value: Difference in accuracy between baseline and negative trials. Accuracy priming values $>0$ stimulus duration. 
indicate that rats performed with higher accuracy in baseline compared to negative trials and hence reflect a negative priming effect.

\section{Accuracy Priming = Baseline Accuracy-NP Accuracy}

It is crucial to distinguish between the phenomenon of negative priming on the one hand and accuracy in the NP task (both during baseline and negative trials) on the other hand. Negative priming does not refer to the accuracy during the negative trials, but to the difference in accuracy between baseline and negative trials - specifically, to lower accuracy in the negative trials as compared to the baseline trials. Groups of animals may therefore exhibit similar accuracy during negative trials, yet differ in their negative priming (if one group has similar accuracy in baseline trials and negative trials, while the other group has significantly higher accuracy in baseline trials compared to negative trials). Likewise, a manipulation may not affect the overall accuracy during negative trials, but still alter negative priming (if it affects the accuracy during baseline trials, and thus the difference in accuracy between baseline and negative trials). This distinction is important for the interpretation of findings in the NP task.

\section{Experiment 1: Effects of isolation rearing on negative visuospatial priming (NP)}

Once stable performance in the task had been reached, both isolates and socials were challenged by decreasing the duration of the prime stimuli from $0.5 \mathrm{~s}$ to $0.25 \mathrm{~s}$. Previous experiments demonstrated that NP in rats is most pronounced when using such challenges. While a trend towards lower accuracy in negative trials versus baseline trials was already observed with the prime stimulus duration used throughout training, robust, statistically significant NP was only observed when rats were challenged with a shorter prime stimulus duration like the one employed here (Amitai et al., 2013). This effect may be due to shorter prime durations favoring processing by preconscious/automatic mechanisms, which may be required for the NP effect. A similar phenomenon is seen in PPI, where shorter intervals between pre-stimulus and stimulus produce maximal PPI values (Braff et al., 1978; Graham, 1975) and have been linked to more automatic, preconscious inhibition, whereas longer intervals are linked to more volitional, consciously controlled inhibition (Swerdlow, Weber, Qu, Light, \& Braff, 2008). In general, stimuli presented below the level of conscious awareness can have a greater impact on response characteristics than stimuli that are consciously perceived (Bargh, 1992; Bornstein, 1989).

After 3 weeks of normal sessions, this short prime duration challenge was repeated for the assessment of reproducibility.

\section{Experiment 2: Effects of amphetamine}

All rats continued to be trained in the NP task and were habituated to the injection procedure with subcutaneous saline injections on the three days before initiation of the experiment. Rats then received $0.25 \mathrm{mg} / \mathrm{kg}$ D-amphetamine (AMPH) or saline 10 min before being tested in the NP task. After a week of drug-free washout, the experiment was repeated. Half of each group of rats (isolates and socials) received AMPH during the first drug challenge and saline during the second challenge, with the other half receiving the injections in the opposite order to minimize confounding order effects. Prime duration was again shortened to $0.25 \mathrm{~s}$ on the drug challenge days. On the washout days between challenges, rats were run in task sessions using standard parameters.

\section{Data analyses}

Pearson Chi square $\left(\chi^{2}\right)$ analysis was performed to compare the number of isolates and socials that reached criterion performance in each experiment.

Accuracy data from Experiment 1 were analyzed using mixed-factor two-way analyses of variance (ANOVA), with Trial Type (baseline or NP) as the within-subjects factor and Rearing (socials or isolates) as the between-subjects factor. Accuracy priming values for both isolates and socials were analyzed using one-sample t-tests to assess whether they were significantly different from 0 . In addition, accuracy priming values and the total number (summed across trial types) of premature responses before the prime and before the probe in isolates and socials were compared using two-tailed t-tests.

Accuracy data from Experiment 2 were analyzed using two-way ANOVA, with the two factors Trial Type (baseline or NP) and Drug Treatment (AMPH or saline). Accuracy priming values for both isolates and socials were analyzed using one-sample t-tests to assess whether they were significantly different from 0 . In addition, the total number of premature responses and ISI responses were compared using two-way ANOVA, with the two factors Drug Treatment (AMPH or saline) and Rearing (socials or isolates).

Bonferroni-corrected post hoc analyses were conducted on primary outcome measures of each group and where significant ANOVAs were observed. The level of significance was set at 0.05 . Data were analyzed using GraphPad Prism ${ }^{\circledR}$ (GraphPad, San Diego, CA) and Sigmaplot ${ }^{\circledR}$ (Systat Software Inc., San Jose, CA, USA).

\section{Results}

Rats were excluded from the study if they were unable to perform the task at a probe stimulus duration of less than $2 \mathrm{~s}$, unable to achieve accuracy above chance levels during 
baseline trials, or if they completed fewer than ten trials of either trial type during the session. As a result, final rat numbers were $\mathrm{n}=23$ socials and $\mathrm{n}=14$ isolates in Experiment 1 (three socials and 15 isolates excluded). When analyzed, this difference in reaching criterion by group was statistically significant (Pearson $\chi^{2}=8.7, p<0.01$ ), indicating fewer isolates attained criterion than socials. For Experiment 2, final rat numbers were $\mathrm{n}=19$ socials and $\mathrm{n}=18$ isolates (seven socials and 11 isolates excluded). When analyzed, this difference to criterion by group was no longer statistically different (Pearson $\chi^{2}=0.4, \mathrm{~ns}$ ), indicating that by this stage, as many isolates attained criterion as socials. The difference in numbers between Experiments 1 and 2 was due to some rats reaching stable criterion performance in time for Experiment 2, but after Experiment 1, while a few other rats' performance dropped below criterion levels after Experiment 1, likely due to the high difficulty and complexity of the task.

\section{Experiment 1: Effects of isolation rearing on NP}

Isolation- and socially reared rats that learned the NP attained criterion at comparable rates. When challenged with a short prime duration, rats performed with lower accuracy during negative trials compared to baseline trials on both challenge days [main effect of Trial Type on accuracy: $F(1,35)=6.9$, $\mathrm{p}<0.05$ during challenge $1, F(1,34)=7.2, \mathrm{p}<0.05$ during challenge 2; see Fig. 3a and b]. Isolates did not differ significantly from socials regarding NP, however, as there was no significant main effect of Rearing $(\mathrm{F}<1, \mathrm{~ns})$ and no Rearing $\times$ Trial Type interaction $(\mathrm{F}<1, \mathrm{~ns})$.

Confirming this pattern, accuracy priming values were significantly different from 0 in both isolates $(\mathrm{p}<0.05)$ and socials $(\mathrm{p}<0.01)$. There was no difference in accuracy priming values between isolates and socials $(\mathrm{F}<1$, ns; see Fig. $3 \mathrm{c})$.

No differences in premature or ISI responses were observed between socials and isolates $(\mathrm{F}<1$, ns, for both measures; data not shown).

\section{Experiment 2: Effects of amphetamine}

A three-way ANOVA of response accuracy detected a significant main effect of Rearing, indicating an overall greater accuracy in isolates $[\mathrm{F}(1,35)=7.2, \mathrm{p}<0.05]$, along with a significant main effect of Trial Type $[\mathrm{F}(1,35)=11.1, \mathrm{p}<0.01]$, and a trend towards a main effect of Drug Treatment $[\mathrm{F}(1,35)=3.2$, $\mathrm{p}=0.086]$ reflecting the overall disruptive effects of amphetamine on NP. Interactions between Rearing $\times$ Trial Type $[\mathrm{F}(1$, $35)=2.0, \mathrm{p}=0.166]$, Rearing $\times$ Drug Treatment $[\mathrm{F}(1,35)=1.9$, $\mathrm{p}=0.179]$, Drug Treatment $\times$ Trial Type $(\mathrm{F}<1, \mathrm{~ns})$, and Rearing $\times$ Drug Treatment $\times$ Trial Type $(\mathrm{F}<1, \mathrm{~ns})$ did not reach statistical significance for accuracy. Considering that

\section{Accuracy}
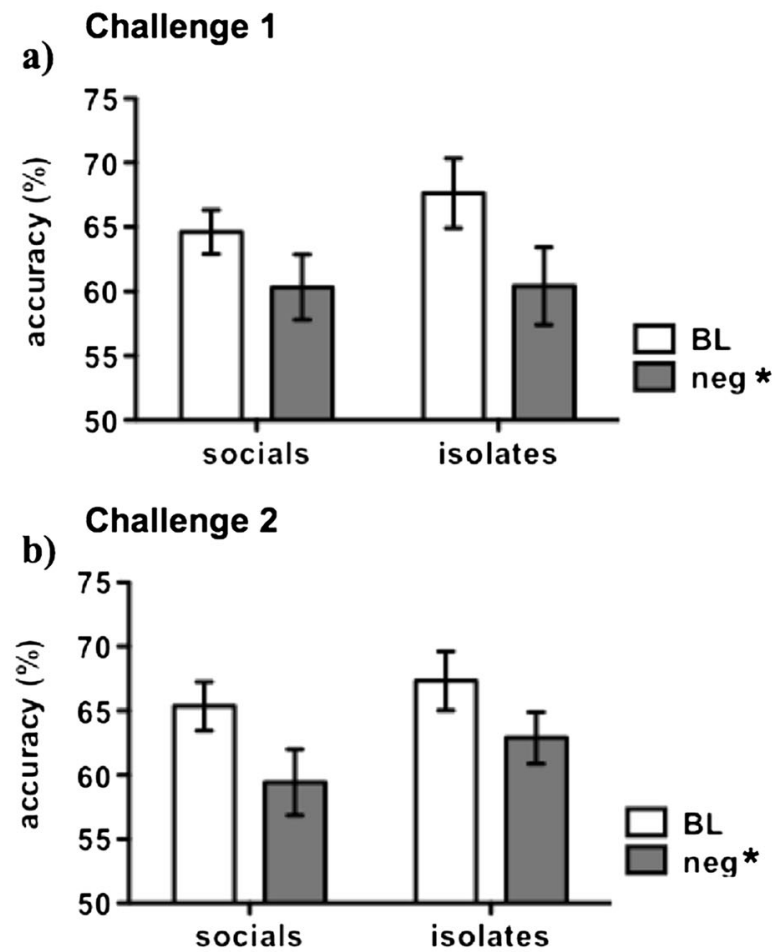

\section{c) Accuracy Priming Values}

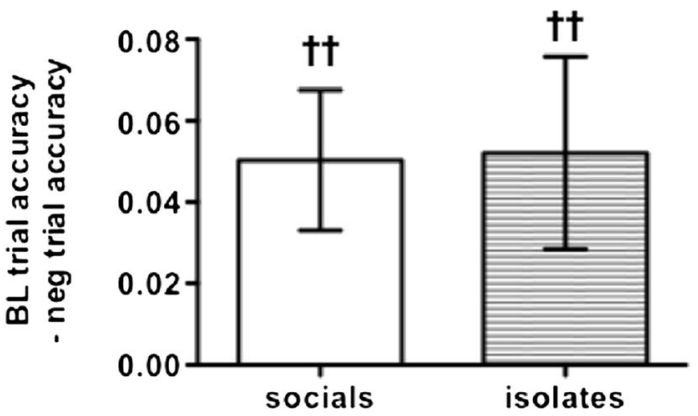

Fig. 3 Negative priming in isolates and socials. Both socials and isolates exhibited negative priming, reflected by lower accuracy in negative trials compared to baseline trials during the first (a) and second (b) short prime challenges. (c) Accuracy priming values were significantly greater than 0 in both socials and isolates, and did not differ between isolates and socials. Values are expressed as mean \pm SEM. Asterisks $\left({ }^{*} p<0.05\right)$ denote a significant main effect of Trial Type; dagger signs ( $\dagger \mathrm{p}<0.05$; $\dagger \dagger \mathrm{p}<0.01$ ) denote a significant difference from $0 . B L$ baseline trials, neg negative trials

without AMPH treatment social and isolates did not differ on NP, but did so after AMPH treatment, and our a priori hypotheses that AMPH treatment would differentially affect socials compared with isolates, we examined the effects of AMPH on each group separately.

Two-way ANOVA of accuracy in socials confirmed that socials continued to perform with lower accuracy during negative trials compared to baseline trials [main effect of Trial Type on accuracy: $F(1,36)=4.6, \mathrm{p}<0.05]$. While ANOVA 
detected no main effect of Drug Treatment $[F(1,36)=1.1$, $\mathrm{p}=0.308]$ and no Drug Treatment $\times$ Trial Type interaction $[F<1, \mathrm{~ns}]$, two-tailed t-tests analyzing saline and AMPH sessions separately found a significant difference in accuracy between trial types only after saline treatment $[t(18)=3.4$, $\mathrm{p}<0.01$ ], with no difference between trial types after AMPH treatment $[t<1$, ns; see Fig. 4a]. In isolates, two-way ANOVA of accuracy also revealed lower accuracy during negative trials compared to baseline trials [main effect of Trial Type on accuracy: $F(1,34)=17.8, \mathrm{p}<0.001]$. In contrast
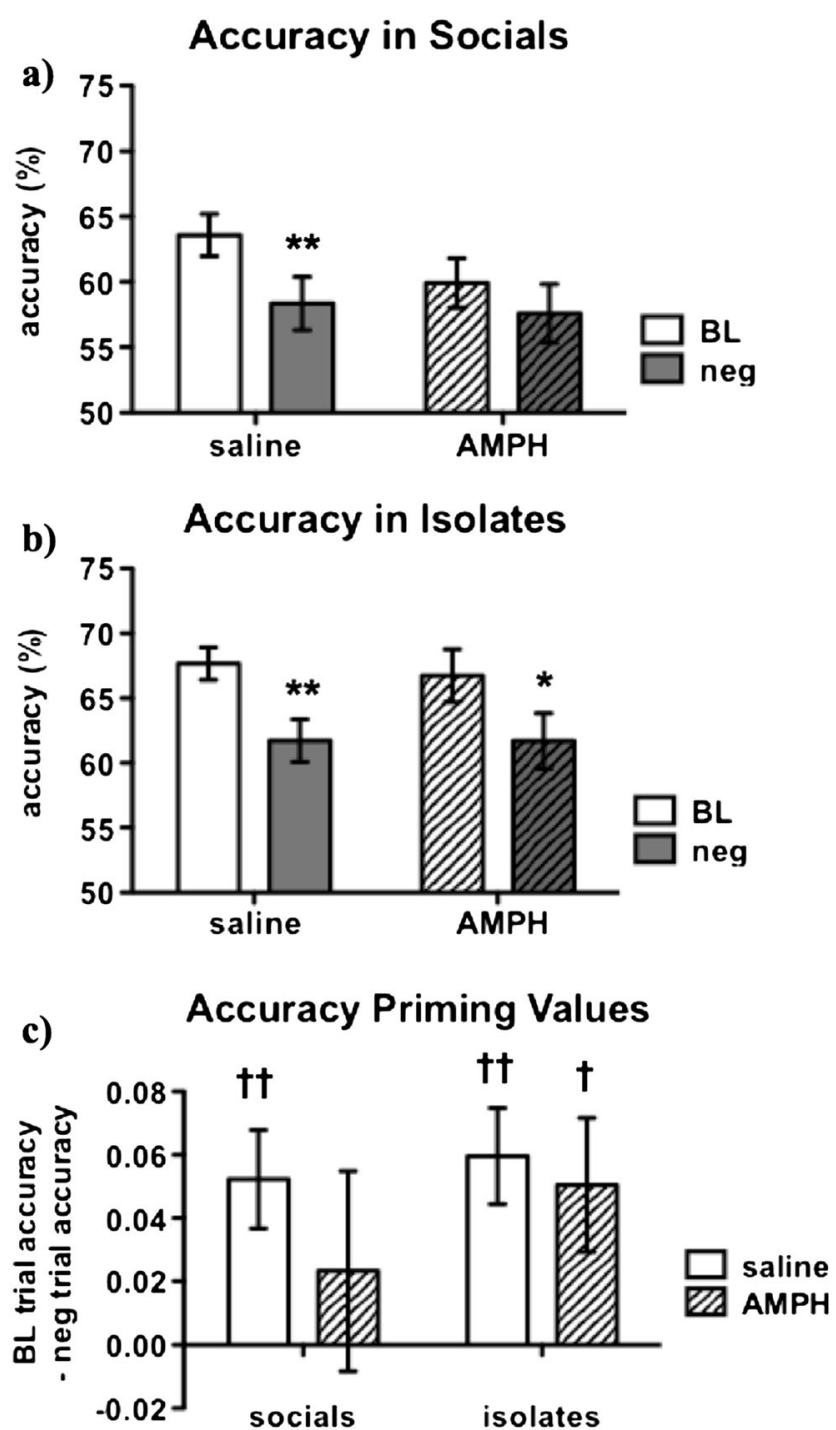

Fig. 4 Effects of D-amphetamine (AMPH) administration on negative priming. (a) Socials exhibited lower accuracy in negative trials compared to baseline trials after saline, but not after AMPH administration. (b) Isolates exhibited lower accuracy in negative trials compared to baseline trials after both saline and AMPH administration. (c) Accuracy priming values were significantly greater than 0 in both socials and isolates after saline administration, but only in isolates after AMPH administration. Values are expressed as mean \pm SEM. Asterisks $(* p<0.05 ; * * p<0.01)$ denote significant differences compared to baseline trials; dagger signs ( $\dagger \mathrm{p}<0.05 ; \dagger \mathrm{p}<0.01)$ denote a significant difference from $0 . B L$ baseline trials, neg negative trials to socials, however, post hoc testing in isolates revealed significant differences between trial types both after saline treatment $[t(17)=3.9, \mathrm{p}<0.01]$ and after AMPH treatment $[t(17)=$ 2.4, $\mathrm{p}<0.05$; see Fig. $4 \mathrm{~b}]$, reflecting sustained NP despite AMPH treatment in isolation reared rats. There was no significant main effect of Drug Treatment $[F<1, \mathrm{~ns}]$ and no Drug Treatment $\times$ Trial Type interaction $[F<1, \mathrm{~ns}]$.

Confirming this pattern, accuracy priming values in socials were significantly different from 0 after saline treatment ( $p<0.01$ ), but not after AMPH administration (ns). In contrast, accuracy priming values for isolates were significantly different from 0 after both saline $(\mathrm{p}<0.01)$ and AMPH $(\mathrm{p}<0.05)$ treatment (see Fig. $4 \mathrm{c}$ ).

AMPH increased premature responses in the NP task [main effect of Drug Treatment: $F(1,35)=24.4, \mathrm{p}<0.001]$. However, a significant main effect of Rearing $[F(1,35)=$ $14.4, \mathrm{p}<0.001]$ and a Rearing $\times$ Drug Treatment interaction $[F(1,35)=9.8, \mathrm{p}<0.01]$ indicated that this effect of AMPH was more pronounced in socials. Post hoc tests confirmed that AMPH treatment increased premature responding only in socials $(\mathrm{p}<0.001)$, not in isolates (ns; see Fig. 5a).

Similarly, ISI responses increased after AMPH administration [main effect of Drug Treatment: $F(1,35)=15.4$,

\section{Premature Responses}

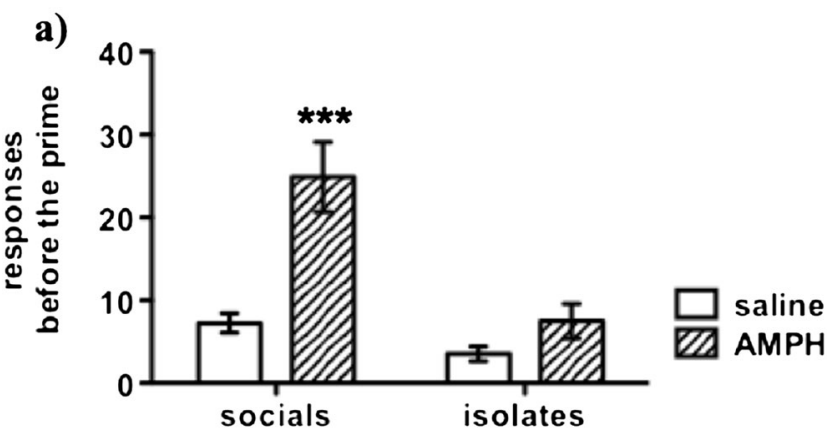

ISI Responses

b)

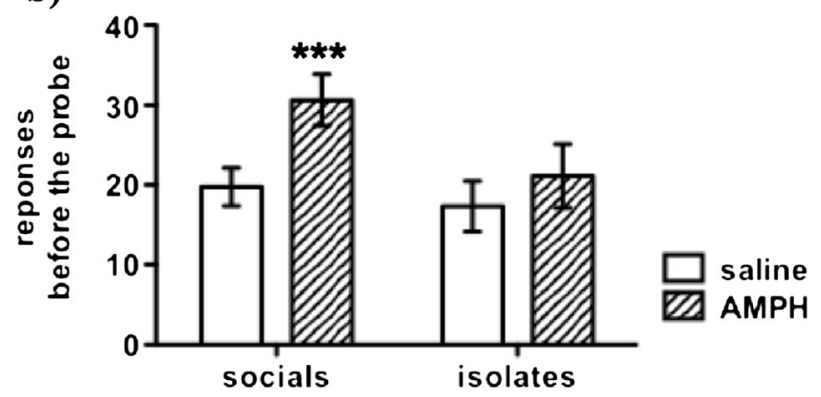

Fig. 5 Effects of D-amphetamine (AMPH) administration on premature and interstimulus interval (ISI) responding. AMPH administration increased premature responses (a) and ISI responses (b) in socials, but not in isolates. Values are expressed as mean \pm SEM. Asterisks $(* * * p<$ $0.001)$ denote significant differences compared to saline administration. $B L$ baseline trials, neg negative trials 
$\mathrm{p}<0.001]$. While there was no main effect of Rearing $[F(1$, $35)=1.9, \mathrm{p}<0.177]$, there was a strong trend towards a Rearing $\times$ Drug Treatment interaction $F(1,35)=4.0$, $\mathrm{p}<0.055]$. Post hoc testing showed that AMPH administration increased ISI responses in socials $(\mathrm{p}<0.001)$, but not in isolates (ns; see Fig. 5b).

\section{Discussion}

In the present study, we demonstrated significant negative visuospatial priming (NP) in a cohort of rats trained in our novel NP task. NP was evidenced by a significant difference in performance during negative versus baseline trials - more specifically, lower accuracy in negative trials than in baseline trials (see Fig. 3a). This finding builds on our initial observations (Amitai et al., 2013) and confirms that this task is capable of reproducibly and quantitatively measuring NP.

Moreover, administration of a low dose $(0.25 \mathrm{mg} / \mathrm{kg})$ of Damphetamine (AMPH) disrupted NP in socially reared rats (socials), as reflected by a loss of difference between baseline and negative trials in AMPH-treated socials (see Fig. 4a). Likewise, accuracy priming values in socials, which were significantly higher than 0 in the saline-only condition, were not significantly different from 0 after AMPH administration (see Fig. 4c), further confirming AMPH-induced NP disruption in these animals. This finding is also consistent with our previous observation (Amitai et al., 2013) that AMPH disrupts NP in animals that have high levels of NP in the drug-free state (as observed in socials in the saline condition in this study).

Notably, both in our previous and in the current study, AMPH disrupted NP not by altering accuracy during negative trials, but by selectively decreasing accuracy in the baseline trials (see Fig. 7b in Amitai et al., 2013, and Fig. 4a in the present study). Theoretically, a manipulation can disrupt NP in a variety of ways - by selectively increasing accuracy in negative trials, by selectively decreasing accuracy in baseline trials, or by increasing or decreasing accuracy in both trial types, but to differing degrees, such that the difference in accuracy between the two trial types is abolished. It is important to note that AMPH did not just lower performance in all trial types (which would have maintained the accuracy difference between baseline and negative trials, and thus maintained NP itself). Likewise, AMPH did not completely wipe out performance in the task, as accuracy in both trial types remained significantly above chance $(33.3 \%$ in a task with three possible response apertures), unlike at higher doses (Amitai et al, 2013). Instead, AMPH primarily eliminated the difference in accuracy between trial types and therefore abolished NP.

While some socials and some isolation-reared rats (isolates) failed to attain criterion performance in the NP task, the numbers of isolates who were unable to acquire the task was notably higher (15 isolates vs. three socials in Experiment
1, 11 isolates vs. seven socials in Experiment 2). This difference was significant for Experiment 1 but not for Experiment 2, suggesting that although isolates exhibited deficient learning, they could eventually learn this complex task. This learning deficit in isolates was likely due to poorer between-session learning (Amitai et al., 2014; Zeeb, Wong, \& Winstanley, 2013). Learning and other cognitive deficits have been observed in isolates in a variety of other tasks, including tests of spatial and working memory (Einon, 1980; Woods, Fiske, \& Ruckelshaus, 1961), reversal learning (Jones, Marsden, \& Robbins, 1991; Krech, Rosenzweig, \& Bennett, 1962; Li, Wu, \& Li, 2007; Powell, et al., 2015; Schrijver, Pallier, Brown, \& Wurbel, 2004), passive avoidance (Valzelli, 1973), recognition memory (Bianchi et al., 2006), and attentional setshifting (Schrijver \& Wurbel, 2001).

Contrary to our expectations, isolates expressed robust NP, and did not differ from socials in this measure (see Fig. 3b). Moreover, in contrast to socials, NP in isolates was not disrupted by the psychostimulant AMPH. Isolates exhibited significant NP both in the saline and in the AMPH condition, reflected by significantly lower accuracy during negative trials compared to baseline trials in both conditions (see Fig. 4b). This finding is confirmed by isolates' accuracy priming values, which were significantly higher than 0 both after saline treatment and after AMPH administration (see Fig. 4c), likewise indicating the presence of NP in both conditions.

Importantly, these same isolates exhibited PPI deficits consistent with previous studies (Amitai et al., 2013), confirming that the isolation procedure was successful in producing neurocognitive deficits, corroborating earlier reports (Cilia et al., 2005; Cilia et al., 2001; Geyer et al., 1993; Varty \& Geyer, 1998; Varty \& Higgins, 1995; Wilkinson et al., 1994) and mirroring deficits found in schizophrenia (Braff et al., 1978; Braff et al., 2001; Grillon, Ameli, Charney, Krystal, \& Braff, 1992). The absence of NP deficits in isolates in our study was unlikely to be due to our isolation rearing procedure being ineffective. Hence, while isolation rearing reproduces a range of behavioral and neurochemical abnormalities found in various developmental and psychiatric disorders, including schizophrenia, it may not be useful for modeling the visuospatial priming deficits seen in these disorders. This finding also suggests that while PPI and NP are both measures of inhibitory processing, they are dissociable and may be mediated by somewhat different substrates and mechanisms.

It is possible that the exclusion of a larger number of isolates due to their inability to reach criterion task performance could have masked a NP deficit in isolates (if the rats excluded due to their inability to learn the tasks were specifically ones that would have exhibited lower levels of NP). It should be noted, though, that accuracy in the task is not necessarily correlated with NP levels (since NP only reflects the difference in accuracy between baseline and negative trials, not overall accuracy levels). Therefore, it cannot automatically 
be concluded that animals excluded due to their poor learning would have necessarily exhibited low NP in the task had their learned it. In addition, when more isolates achieved criterion in Experiment 2, NP still did not differ between groups. Furthermore, those rats that did achieve NP criterion maintained a decreased PPI compared to socially reared rats, supporting the separation of PPI versus NP effects of this manipulation.

It remains possible that this NP task, in its current form, cannot detect increases in NP beyond a certain point, and thus may not be able to detect greater NP in socials due to ceiling effects. If this is the case, possible NP deficits in isolates may have been missed in the present study. Such ceiling effects may have also prevented the detection of possible enhancement of NP after AMPH administration in isolates. Future studies will determine whether further optimizing of the NP task may be required to detect and interpret the effects of behavioral and pharmacological manipulations on NP.

In addition to AMPH disrupting NP in socials, but not isolates, isolates also did not exhibit the AMPH-induced effects on motor impulsivity seen in socials in this task. AMPH increased premature responding and responding during the inter-stimulus interval (ISI) in socials, but not isolates (Fig. 5).

The fact that AMPH increased responding during the ISI in socials, but not isolates, raises the hypothetical situation that accuracy during probe trials (and thus NP) could have been affected if animals were responding in the same location throughout the ISI and into the probe trial. In brief, responses during the ISI are most likely to occur in the location that displayed the target stimulus during the prime trial. Increasing such responses would have the same effect on baseline and negative trials (given that in both trial types, the target stimulus will appear in a different location in the probe trial than where it was located in the prime trial), and would thus not affect NP. If in the unlikely condition, however, rats tended to perform ISI responses into the location that contained the non-target stimulus during the prime trial, this could have a trial-type selective effect. In negative trials, the target in the probe trial appears in the location that had contained the non-target stimulus in the prime trial; rats that were performing persistent ISI responses into this location would increase their accuracy if these ISI responses "carried over" into the probe trial. Conversely, during baseline trials, the target stimulus in the probe trial appears in the location that contained neither the target nor the non-target stimulus during the prime trial; ISI responses persevering in the non-target location would result in lower accuracy if they continued into the probe trial. In this way, ISI responses into the initial nontarget location could selectively decrease accuracy in baseline trials, increase accuracy in negative trials, and thus reduce NP. Therefore, hypothetically, socials may exhibit a disruption of NP during AMPH exposure as an indirect result of increased
ISI responses, and isolates may have preserved NP because they do not experience this increase in ISI responses.

It should be noted that this hypothetical scenario is highly unlikely as ISI responses rarely occurred preferentially into the non-target stimulus location during the prime trial. Indeed, the very fact that NP is observed in this task (i.e., that rats tend to be less accurate in negative trials) suggests that rats learn to ignore the non-target stimulus during the prime trial, and to suppress responding in its location. Having to overcome that inhibition in order to correctly respond when, during a negative trial, this location then goes on to display the target stimulus, is what presumably leads to the decreased accuracy in negative trials as compared to baseline trials and thus NP. Nonetheless, to fully rule out the possibility that rats somehow still preferentially perform ISI responses into the location displaying the non-target location in the prime trial, future experiments could explicitly track the location into which ISI responses occur.

Taken together, the observations that AMPH disrupted NP and increased premature and ISI responses in socials, but not isolates, suggest a behavioral resistance to the psychostimulant effects of AMPH in isolates. It must be noted, however, that no definitive conclusions regarding hypoversus hypersensitivity to the effects of AMPH in isolates can be drawn based on the present study, since only one dose of AMPH was used.

Interestingly, earlier studies have reported increased behavioral sensitivity to the effects of AMPH and other psychostimulants in isolation-reared animals tested in other tasks. Rats reared in isolation have been found to be hypersensitive to AMPH-induced hyperlocomotion (Garzon, Fuentes, \& Del Rio, 1979; Jones et al., 1992; Jones et al., 1990), but see (Bowling \& Bardo, 1994), stereotypies (Jones et al., 1992; Sahakian et al., 1975), and increased responding for a conditioned reinforcer (Jones et al., 1990). Locomotor activation due to cocaine administration was also found to be potentiated in isolates (Phillips et al., 1994). For more complex behaviors, however, resistance to psychostimulantinduced disruptive effects has been reported that is consistent with the findings of this study. For example, in socials, but not isolates, AMPH increased premature responses in the fivechoice serial reaction time task (Baarendse, Counotte, O'Donnell, \& Vanderschuren, 2013; Dalley, Theobald, Pereira, Li, \& Robbins, 2002) and disrupted performance of a gambling task (Zeeb et al., 2013). Thus, psychostimulant sensitivity in isolates versus socials may differ depending upon the behavior examined. It must be noted that these comparison should be viewed with some caution as the studies differ regarding amphetamine dose, administration route (subcutaneous, intraperitoneal, intravenous, or intracerebral), and type of psychostimulant used (amphetamine vs. cocaine). In particular, the studies of locomotor function and stereotypies where evidence of amphetamine hypersensitivity were found 
all used higher doses of amphetamine (Garzon et al., 1979; Jones et al., 1992; Jones et al., 1990; Sahakian et al., 1975) or cocaine (Phillips et al., 1994). This is likely due to the fact that simple locomotor tests are less sensitive to disruption by psychostimulants and nonspecific motor effects than more complex behavioral and cognitive tasks, and that even higher doses of psychostimulants are needed to evoke stereotyped behaviors.

Furthermore, the simple versus complex behaviors described above are mediated by different brain systems and the altered psychostimulant sensitivity of specific behaviors in isolates may reflect differing effects of isolation rearing on the neural substrates that regulate these behaviors. Subcortical DA activity, especially in the striatum, appears to be both enhanced in the drug-free state and hypersensitive to psychostimulants in isolates (Hall, 1998; Howes, Dalley, Morrison, Robbins, \& Everitt, 2000; Jones et al., 1992). This striatal hypersensitivity contrasts with findings in the prefrontal cortex (PFC), with several studies reporting decreased DA activity in this region in isolates (Blanc et al., 1980; Hall, 1998; Heidbreder et al., 2000; Robbins et al., 1996). Isolation-reared animals also exhibit decreased PFC volume (Day-Wilson, Jones, Southam, Cilia, \& Totterdell, 2006; Silva-Gomez, Rojas, Juarez, \& Flores, 2003), along with reduced dendritic arborization in the PFC (Pascual, Zamora-León, \& Valero-Cabré, 2006; Silva-Gomez et al., 2003). It is possible that isolation rearing has opposite effects on DA neurotransmission in the PFC and the striatum; cortical and subcortical DA projections often show reciprocal changes in activity (Pycock et al., 1980). Indeed, activation of cortical DA transmission can suppress DA release in subcortical areas, while, conversely, reduction of DA activity in cortical regions can disinhibit subcortical DA release (Deutch, 1993).

The pattern outlined above exhibits similarities with the updated and most widely accepted version of the dopamine hypothesis of schizophrenia, which proposes hyperdopaminergic activity in subcortical regions but hypodopaminergic activity in cortical regions (Davis et al., 1991). AMPH-stimulated striatal DA release is increased in schizophrenia ( Abi-Dargham et al., 1998; Breier et al., 1997; Laruelle et al., 1996). Less is known, however, about AMPHinduced DA release in extrastriatal regions, including the PFC, in these patients. Hypoactivation of the PFC in schizophrenia has been documented in numerous studies (Buchsbaum, 1990; Davidson \& Heinrichs, 2003; Harrison, 1999), along with volume reductions in frontal cortical areas (Harrison, 1999; Kurachi, 2003; Shenton, Dickey, Frumin, \& McCarley, 2001). Positron emission tomography studies reported by Abi-Dargham and Moore (2003) show regionally specific upregulation of D1 DA receptors in the prefrontal cortex of schizophrenia patients; additional results from clinical trials indicate that this finding most likely represents a compensatory but insufficient response to a chronic deficit in presynaptic DA function in the PFC (Abi-Dargham \& Moore, 2003). Moreover, while psychostimulants like AMPH exacerbate positive symptoms of schizophrenia such as hallucinations and delusions, in a manner correlated with DA release (Laruelle et al., 1999), AMPH has been found to ameliorate the cognitive schizophrenia symptoms and improve the performance of schizophrenia patients in frontalmediated cognitive tasks (Pietrzak, Snyder, \& Maruff, 2010). It should be noted that while the latter finding is of great interest for its implications for the role of DA transmission in cognitive schizophrenia symptoms, AMPH's putative detrimental effects on the positive symptoms of these patients may limit its therapeutic potential - certainly controlled testing would be required.

Both the PFC (Wright et al., 2006; Wright, McMullin, Martis, Fischer, \& Rauch, 2005) and dysfunction of dopaminergic signaling (Swerdlow et al., 1997; Wylie \& Stout, 2002; Yamaguchi \& Kobayashi, 1998) have been strongly implicated in the mediation of visuospatial priming in humans. While no microinfusion or microdialysis studies have been conducted to date in rodents undergoing the NP task that could indicate the neural substrates and neurotransmitters involved, it is not unreasonable to hypothesize that the PFC and dopaminergic signaling play a role in visuospatial priming in rats also. AMPH significantly increases PFC DA release in rats (Moghaddam \& Bunney, 1989). We have previously found that AMPH had a rate-dependent effect on NP in rats, increasing NP in animals with low levels of NP in the drug-free state and, conversely, disrupting NP in those with high levels of NP in the drug-free state (Amitai et al., 2013). Optimal DA transmission levels in the PFC may therefore be required for maximum levels of PFC-controlled functions (Mattay et al., 2000; Mattay et al., 2003; Mehta et al., 2000), including visuospatial priming.

The above considerations suggest that isolation rearinginduced reductions in DA responsivity in the PFC might account for the observation of the attenuated AMPH effects on NP in isolates compared with socials. Although NP was not disrupted in isolates and AMPH did not improve NP in these animals, as we had hypothesized, altered sensitivity to AMPH was observed in isolates at a dose that impaired NP in socials. These findings implicate altered PFC function in isolationreared rats, which would suggest these rats may still replicate some aspects of schizophrenia.

While direct AMPH effects on the DA system may represent the most parsimonious explanation for the differential response to AMPH in isolates when compared to socials, it is conceivable that some of the observed effects may be explained a by differences in serotonin (5-HT) signaling. Increased 5-HT release in the PFC is associated with higher levels of premature responding in a test of visuospatial attention (Dalley, Theobald, Eagle, Passetti, \& Robbins, 2002), and PFC 5-HT efflux was elevated during rats' performance of an 
impulsive choice task (Winstanley, Theobald, Dalley, Cardinal, \& Robbins, 2006). In isolation-reared animals, the effect of various challenges (AMPH administration, footshock, conditioned fear, potassium chloride administration, or exposure to a novel environment) to increase 5-HT release is enhanced in the NAcc (Fulford \& Marsden, 1998), but attenuated in the PFC (Bickerdike, Wright, \& Marsden, 1993; Dalley, Theobald, Pereira et al., 2002). Given the central role of PFC 5-HT in motor impulsivity (Evenden, 1999; Evenden, 1999; Mobini, Chiang, Ho, Bradshaw, \& Szabadi, 2000; Soubrié, 1986) and specifically in premature responses (Puumala \& Sirviö, 1998), it is plausible that reduced 5-HT responsivity in the PFC may also contribute to the attenuation of AMPH effects on premature responses observed in this study. Notably, a study investigating the performance of isolates in a test of visuospatial attention found that they exhibited resistance to AMPH-induced increases in premature responding that was associated with attenuated 5-HT release in the PFC after AMPH challenge, while AMPH-induced DA release in the PFC was unaffected (Dalley, Theobald, Pereira et al., 2002).

In conclusion, the present findings replicate and expand our previous report that NP can be measured in rats and is sensitive to disruption by AMPH. In contrast with our hypothesis, however, isolation rearing did not disrupt negative visuospatial priming in rats, despite inducing PPI deficits consistent with various developmental and psychiatric disorders characterized by impaired information processing, including schizophrenia. Furthermore, and in contrast with socially reared rats, isolation-reared rats did not exhibit disruptions of NP and increases in premature responses in this task in response to AMPH administration. This phenomenon may reflect reduced monoaminergic activity in the PFC, but further research is needed to more firmly establish the neurochemical underpinnings of the observations in this study.

Acknowledgments This work was supported by NIH grant R01MH104344, R01-MH059803, R01-MH042228, R21-MH091571 and the Veteran's Administration VISN 22 Mental Illness Research, Education, and Clinical Center, as well as the Tourette's Syndrome Association. The authors would like to thank Dr. Mark Geyer for his advice and support, and Ms. Mahalah Buell and Mr. Richard F. Sharp for their assistance.

\section{References}

Abi-Dargham, A., Gil, R., Krystal, J., Baldwin, R. M., Seibyl, J. P., Bowers, M., van Dyck, C. H., Carney, D. S., Innis, R. B., \& Laruelle M. (1998). Increased striatal dopamine transmission in schizophrenia: Confirmation in a second cohort. The American Journal of Psychiatry, 155(6), 761-767.

Abi-Dargham, A., \& Moore, H. (2003). Prefrontal DA transmission at D1 receptors and the pathology of schizophrenia. The Neuroscientist, 9(5), 404-416.
Agid, O., Shapira, B., Zislin, J., Ritsner, M., Hanin, B., Murad, H., Troudart, T., Bloch, M., Heresco-Levy, U., \& Lerer, B. (1999). Environment and vulnerability to major psychiatric illness: A case control study of early parental loss in major depression, bipolar disorder and schizophrenia. Molecular Psychiatry, 4(2), 163-172.

Amitai, N., Weber, M., Swerdlow, N. R., Sharp, R. F., Breier, M. R., Halberstadt, A. L., \& Young, J. W. (2013). A novel visuospatial priming task for rats with relevance to Tourette syndrome and modulation of dopamine levels. Neuroscience and Biobehavioral Reviews, 37(6), 1139-1149.

Amitai, N., Young, J. W., Higa, K., Sharp, R. F., Geyer, M. A., \& Powell, S. B. (2014). Isolation rearing effects on probabilistic learning and cognitive flexibility in rats. Cognitive, Affective, \& Behavioral Neuroscience, 14(1), 388-406.

Baarendse, P. J., Counotte, D. S., O'Donnell, P., \& Vanderschuren, L. J. (2013). Early social experience is critical for the development of cognitive control and dopamine modulation of prefrontal cortex function. Neuropsychopharmacology, 38(8), 1485-1494.

Bakshi, V. P., \& Geyer, M. A. (1999). Ontogeny of isolation rearinginduced deficits in sensorimotor gating in rats. Physiology and Behavior, 67(3), 385-392.

Bakshi, V. P., Swerdlow, N. R., Braff, D. L., \& Geyer, M. A. (1998). Reversal of isolation rearing-induced deficits in prepulse inhibition by Seroquel and olanzapine. Biological Psychiatry, 43(6), 436-445.

Bargh, J. A. (1992). The ecology of automaticity: Toward establishing the conditions needed to produce automatic processing effects. American Journal of Psychology, 105(2), 181-199.

Beech, A., Powell, T., McWilliam, J., \& Claridge, G. (1989). Evidence of reduced 'cognitive inhibition' in schizophrenia. British Journal of Clinical Psychology, 28(Pt 2), 109-116.

Bianchi, M., Fone, K. F., Azmi, N., Heidbreder, C. A., Hagan, J. J., \& Marsden, C. A. (2006). Isolation rearing induces recognition memory deficits accompanied by cytoskeletal alterations in rat hippocampus. European Journal of Neuroscience, 24(10), 2894-2902.

Bickerdike, M. J., Wright, I. K., \& Marsden, C. A. (1993). Social isolation attenuates rat forebrain 5-HT release induced by KCI stimulation and exposure to a novel environment. Behavioral Pharmacology, 4(3), 231-236.

Blanc, G., Herve, D., Simon, H., Lisoprawski, A., Glowinski, J., \& Tassin, J. P. (1980). Response to stress of mesocortico-frontal dopaminergic neurones in rats after long-term isolation. Nature, 284(5753), 265-267.

Bornstein, R. (1989). Exposure and affect: Overview and meta-analysis of research, 1968-1987. Psychological Bulletin, 106(2), 265-289.

Bowling, S. L., \& Bardo, M. T. (1994). Locomotor and rewarding effects of amphetamine in enriched, social, and isolate reared rats. Pharmacology Biochemistry and Behavior, 48(2), 459-464.

Braff, D. L. (1993). Information processing and attention dysfunctions in schizophrenia. Schizophrenia Bulletin, 19(2), 233-259.

Braff, D. L., Geyer, M. A., \& Swerdlow, N. R. (2001). Human studies of prepulse inhibition of startle: Normal subjects, patient groups, and pharmacological studies. Psychopharmacology, 156(2-3), 234 258.

Braff, D., Stone, C., Callaway, E., Geyer, M., Glick, I., \& Bali, L. (1978). Prestimulus effects on human startle reflex in normals and schizophrenics. Psychophysiology, 15(4), 339-343.

Breier, A., Malhotra, A. K., Pinals, D. A., Weisenfeld, N. I., \& Pickar, D. (1997). Association of ketamine-induced psychosis with focal activation of the prefrontal cortex in healthy volunteers. The American Journal of Psychiatry, 154, 805-811.

Buchanan, R. W., Holstein, C., \& Breier, A. (1994). The comparative efficacy and long-term effect of clozapine treatment on neuropsychological test performance. Biological Psychiatry, 36, 717-725.

Buchsbaum, M. S. (1990). The frontal lobes, basal ganglia, and temporal lobes as sites for schizophrenia. Schizophrenia Bulletin, 16(3), 379-389. 
Cilia, J., Hatcher, P. D., Reavill, C., \& Jones, D. N. (2005). Long-term evaluation of isolation-rearing induced prepulse inhibition deficits in rats: An update. Psychopharmacology, 180(1), 57-62.

Cilia, J., Reavill, C., Hagan, J. J., \& Jones, D. N. (2001). Long-term evaluation of isolation-rearing induced prepulse inhibition deficits in rats. Psychopharmacology, 156(2-3), 327-337.

Dalley, J. W., Theobald, D. E., Eagle, D. M., Passetti, F., \& Robbins, T. W. (2002a). Deficits in impulse control associated with tonicallyelevated serotonergic function in rat prefrontal cortex. Neuropsychopharmacology, 26(6), 716-728.

Dalley, J. W., Theobald, D. E., Pereira, E. A., Li, P. M., \& Robbins, T. W. (2002b). Specific abnormalities in serotonin release in the prefrontal cortex of isolation-reared rats measured during behavioural performance of a task assessing visuospatial attention and impulsivity. Psychopharmacology, 164(3), 329-340.

Davidson, L. L., \& Heinrichs, R. W. (2003). Quantification of frontal and temporal lobe brain-imaging findings in schizophrenia: A metaanalysis. Psychiatry Research, 122(2), 69-87.

Davis, K. L., Kahn, R. S., Ko, G., \& Davidson, M. (1991). Dopamine in schizophrenia: A review and reconceptualization. The American Journal of Psychiatry, 148(11), 1474-1486.

Day-Wilson, K. M., Jones, D. N., Southam, E., Cilia, J., \& Totterdell, S. (2006). Medial prefrontal cortex volume loss in rats with isolation rearing-induced deficits in prepulse inhibition of acoustic startle. Neuroscience, 141(3), 1113-1121.

Deutch, A. Y. (1993). Prefrontal cortical dopamine systems and the elaboration of functional corticostriatal circuits: Implications for schizophrenia and Parkinson's disease. Journal of Neural Transmission. General Section, 91(2-3), 197-221.

Einon, D. (1980). Spatial memory and response strategies in rats: Age, sex and rearing differences in performance. Quarterly Journal of Experimental Psychology, 32(3), 473-489.

Einon, D. F., \& Morgan, M. J. (1977). A critical period for social isolation in the rat. Developmental Psychobiology, 10(2), 123-132.

Elkins, I. J., \& Cromwell, R. L. (1994). Priming effects in schizophrenia: Associative interference and facilitation as a function of visual context. Journal of Abnormal Psychology, 103(4), 791-800.

Evenden, J. (1999a). Impulsivity: A discussion of clinical and experimental findings. Journal of Psychopharmacology, 13(2), 180-192.

Evenden, J. L. (1999b). Varieties of impulsivity. Psychopharmacology, 146, 348-361.

Fone, K. C., \& Porkess, M. V. (2008). Behavioural and neurochemical effects of post-weaning social isolation in rodents-relevance to developmental neuropsychiatric disorders. Neuroscience and Biobehavioral Reviews, 32(6), 1087-1102.

Fulford, A. J., \& Marsden, C. A. (1998). Effect of isolation-rearing on conditioned dopamine release in vivo in the nucleus accumbens of the rat. Journal of Neurochemistry, 70(1), 384-390.

Garzon, J., Fuentes, J. A., \& Del Rio, J. (1979). Antidepressants selectively antagonize the hyperactivity induced in rats by long-term isolation. European Journal of Pharmacology, 59(3-4), 293-296.

Geyer, M. A., Wilkinson, L. S., Humby, T., \& Robbins, T. W. (1993). Isolation rearing of rats produces a deficit in prepulse inhibition of acoustic startle similar to that in schizophrenia. Biological Psychiatry, 34(6), 361-372.

Graham, F. K. (1975). Presidential address, 1974. The more or less startling effects of weak prestimulation. Psychophysiology, 12(3), 238248.

Grillon, C., Ameli, R., Charney, D. S., Krystal, J., \& Braff, D. (1992). Startle gating deficits occur across prepulse intensities in schizophrenic patients. Biological Psychiatry, 32(10), 939-943.

Hall, F. S. (1998). Social deprivation of neonatal, adolescent, and adult rats has distinct neurochemical and behavioral consequences. Critical Reviews in Neurobiology, 12(1-2), 129-162.

Harrison, P. J. (1999). The neuropathology of schizophrenia. A critical review of the data and their interpretation. Brain, 122(Pt 4), 593-624.
Harvey, P. D., Rabinowitz, J., Eerdekens, M., \& Davidson, M. (2005). Treatment of cognitive impairment in early psychosis: A comparison of risperidone and haloperidol in a large long-term trial. The American Journal of Psychiatry, 162(10), 1888-1895.

Heidbreder, C. A., Weiss, I. C., Domeney, A. M., Pryce, C., Homberg, J., Hedou, G., Feldon, J., Moran, M. C., \& Nelson, P. (2000). Behavioral, neurochemical and endocrinological characterization of the early social isolation syndrome. Neuroscience, 100(4), 749-768.

Howes, S. R., Dalley, J. W., Morrison, C. H., Robbins, T. W., \& Everitt, B. J. (2000). Leftward shift in the acquisition of cocaine self-administration in isolation-reared rats: Relationship to extracellular levels of dopamine, serotonin and glutamate in the nucleus accumbens and amygdalastriatal FOS expression. Psychopharmacology, 151(1), 55-63.

Jibson, M. D., \& Tandon, R. (1998). New atypical antipsychotic medications. Journal of Psychiatric Research, 32(3-4), 215-228.

Jones, G. H., Hernandez, T. D., Kendall, D. A., Marsden, C. A., \& Robbins, T. W. (1992). Dopaminergic and serotonergic function following isolation rearing in rats: Study of behavioural responses and postmortem and in vivo neurochemistry. Pharmacology Biochemistry and Behavior, 43(1), 17-35.

Jones, G. H., Marsden, C. A., \& Robbins, T. W. (1990). Increased sensitivity to amphetamine and reward-related stimuli following social isolation in rats: Possible disruption of dopamine-dependent mechanisms of the nucleus accumbens. Psychopharmacology, 102(3), 364-372.

Jones, G. H., Marsden, C. A., \& Robbins, T. W. (1991). Behavioural rigidity and rule-learning deficits following isolation-rearing in the rat: Neurochemical correlates. Behavioural Brain Research, 43(1), 35-50.

Krech, D., Rosenzweig, M. R., \& Bennett, E. L. (1962). Relations between chemistry and problem-solving among rats raised in enriched and impoverished environments. Journal of Comparative Physiology and Psychology, 55, 801-807.

Kumari, V., \& Gray, J. A. (1999). Smoking withdrawal, nicotine dependence and prepulse inhibition of the acoustic startle reflex. Psychopharmacology, 141(1), 11-15.

Kurachi, M. (2003). Pathogenesis of schizophrenia: Part I. Symptomatology, cognitive characteristics and brain morphology. Psychiatry and Clinical Neurosciences, 57(1), 3-8.

Lapiz, M. D., Fulford, A., Muchimapura, S., Mason, R., Parker, T., \& Marsden, C. A. (2003). Influence of postweaning social isolation in the rat on brain development, conditioned behavior, and neurotransmission. Neuroscience and Behavioral Physiology, 33(1), 13-29.

Laruelle, M., Abi-Dargham, A., Gil, R., Kegleles, L., \& Innis, R. (1999). Increased dopamine transmission in schizophrenia: relationship to illness phases. Biological Psychiatry, 46(1),56-72.

Laruelle, M., Abi-Dargham, A., van Dyck, C. H., Gil, R., D'Souza, C. D., Erdos, J., McCance, E., Rosenblatt, W., Fingado, C., Zoghbi, S. S., Baldwin, R. M., Seibyl, J. P., Krystal, J. H., Charney, D. S., \& Innis, R. B. (1996). Single photon emission computerized tomography imaging of amphetamine-induced dopamine release in drug-free schizophrenic subjects. Proceedings of the National Academy of Sciences of the United States of America, 93(17), 9235-9240.

Li, N., Wu, X., \& Li, L. (2007). Chronic administration of clozapine alleviates reversal-learning impairment in isolation-reared rats. Behavioral Pharmacology, 18(2), 135-145.

Lim, C., Chong, S. A., \& Keefe, R. S. (2009). Psychosocial factors in the neurobiology of schizophrenia: A selective review. Annals of the Academy of Medicine, Singapore, 38(5), 402-406.

MacQueen, G. M., Galway, T., Goldberg, J. O., \& Tipper, S. P. (2003). Impaired distractor inhibition in patients with schizophrenia on a negative priming task. Psychological Medicine, 33(1), 121-129.

Mattay, V. S., Callicott, J. H., Bertolino, A., Heaton, I., Frank, J. A., Coppola, R., Berman, K. F., Goldberg, T. W., \& Weinberger, D. R. (2000). Effects of dextroamphetamine on cognitive performance and cortical activation. NeuroImage, 12(3), 268-275.

Mattay, V. S., Goldberg, T. E., Fera, F., Hariri, A. R., Tessitore, A., Egan, M. F., Kolachana, B., Callicott, J. H., \& Weinberger, D. R. (2003). 
Catechol O-methyltransferase val158-met genotype and individual variation in the brain response to amphetamine. Proceedings of the National Academy of Sciences of the United States of America, 100(10), 6186-6191.

Mehta, M. A., Owen, A. M., Sahakian, B. J., Mavaddat, N., Pickard, J. D., \& Robbins, T. W. (2000). Methylphenidate enhances working memory by modulating discrete frontal and parietal lobe regions in the human brain. Journal of Neuroscience, 20(6), RC65.

Meltzer, H. Y. (2002). Suicidality in schizophrenia: A review of the evidence for risk factors and treatment options. Current Psychiatry Reports, 4(4), 279-283.

Meltzer, H. Y., \& McGurk, S. R. (1999). The effects of clozapine, risperidone, and olanzapine on cognitive function in schizophrenia. Schizophrenia Bulletin, 25(2), 233-255.

Mobini, S., Chiang, T. J., Ho, M. Y., Bradshaw, C. M., \& Szabadi, E. (2000). Effects of central 5-hydroxytryptamine depletion on sensitivity to delayed and probabilistic reinforcement. Psychopharmacology, 152, 390-397.

Moghaddam, B., \& Bunney, B. S. (1989). Differential effect of cocaine on extracellular dopamine levels in rat medial prefrontal cortex and nucleus accumbens: Comparison to amphetamine. Synapse, 4(2), 156-161.

Mortimer, A. M. (1997). Cognitive function in schizophrenia: Do neuroleptics make a difference? Pharmacology Biochemistry and Behavior, 56(4), 789-795.

Nuechterlein, K. H., \& Dawson, M. E. (1984). Information processing and attentional functioning in the developmental course of schizophrenic disorders. Schizophrenia Bulletin, 10, 160-203.

Pascual, R., Zamora-León, S. P., \& Valero-Cabré, A. (2006). Effects of postweaning social isolation and re-socialization on the expression of vasoactive intestinal peptide (VIP) and dendritic development in the medial prefrontal cortex of the rat. Acta Neurobiologiae Experimentalis, 66(1), 7-14.

Phillips, G. D., Howes, S. R., Whitelaw, R. B., Wilkinson, L. S., Robbins, T. W., \& Everitt, B. J. (1994). Isolation rearing enhances the locomotor response to cocaine and a novel environment, but impairs the intravenous self-administration of cocaine. Psychopharmacology, $115(3), 407-418$.

Pietrzak, R. H., Snyder, P. J., \& Maruff, P. (2010). Amphetamine-related improvement in executive function in patients with chronic schizophrenia is modulated by practice effects. Schizophrenia Research, 124(1-3), 176-182.

Potkin, S. G., Fleming, K., Jin, Y., \& Gulasekaram, B. (2001). Clozapine enhances neurocognition and clinical symptomatology more than standard neuroleptics. Journal of Clinical Psychopharmacology, 21(5), 479-483.

Powell, S. B. (2010). Models of neurodevelopmental abnormalities in schizophrenia. Current Topics in Behavioral Neurosciences, 4, 435-481.

Powell, S. B., Khan, A., Young, J. W., Scott, C. N., Buell, M. R., Caldwell, S., et al. (2015). Early adolescent emergence of reversal learning impairments in isolation-reared rats. Dev Neurosci, in press.

Puumala, T., \& Sirviö, J. (1998). Changes in activities of dopamine and serotonin systems in the frontal cortex underlie poor choice accuracy and impulsivity of rats in an attention task. Neuroscience, 83(2), 489-499.

Pycock, C. J., Kerwin, R. W., \& Carter, C. J. (1980). Effect of lesion of cortical dopamine terminals on subcortical dopamine receptors in rats. Nature, 286(5768), 74-76.

Robbins, T. W., Jones, G. H., \& Wilkinson, L. S. (1996). Behavioural and neurochemical effects of early social deprivation in the rat. Journal of Psychopharmacology, 10(1), 39-47.

Sahakian, B. J., Robbins, T. W., Morgan, M. J., \& Iversen, S. D. (1975). The effects of psychomotor stimulants on stereotypy and locomotor activity in socially-deprived and control rats. Brain Research, 84(2), 195-205.

Schrijver, N. C., Pallier, P. N., Brown, V. J., \& Wurbel, H. (2004). Double dissociation of social and environmental stimulation on spatial learning and reversal learning in rats. Behavioural Brain Research, 152(2), 307-314.
Schrijver, N. C., \& Wurbel, H. (2001). Early social deprivation disrupts attentional, but not affective, shifts in rats. Behavioral Neuroscience, $115(2), 437-442$.

Shenton, M. E., Dickey, C. C., Frumin, M., \& McCarley, R. W. (2001). A review of MRI findings in schizophrenia. Schizophrenia Research, $49(1-2), 1-52$.

Silva-Gomez, A. B., Rojas, D., Juarez, I., \& Flores, G. (2003). Decreased dendritic spine density on prefrontal cortical and hippocampal pyramidal neurons in postweaning social isolation rats. Brain Research, 983(1-2), 128-136.

Soubrié, P. (1986). Serotonergic neurons and behavior. Journal de Pharmacologie, 17(2), 107-112.

Swerdlow, N. R., Hartman, P. L., \& Auerbach, P. P. (1997). Changes in sensorimotor inhibition across the menstrual cycle: Implications for neuropsychiatric disorders. Biological Psychiatry, 41(4), 452-460.

Swerdlow, N. R., Weber, M., Qu, Y., Light, G. A., \& Braff, D. L. (2008). Realistic expectations of prepulse inhibition in translational models for schizophrenia research. Psychopharmacology, 199(3), 331-388.

Tipper, S. P. (1985). The negative priming effect: Inhibitory priming by ignored objects. The Quarterly Journal of Expiremental Psychology A, 37(4), 571-590.

Tipper, S. P. (2001). Does negative priming reflect inhibitory mechanisms? A review and integration of conflicting views. The Quarterly Journal of Expiremental Psychology A, 54(2), 321-343.

Valzelli, L. (1973). The "isolation syndrome" in mice. Psychopharmacologia, 31(4), 305-320.

Varty, G. B., \& Geyer, M. A. (1998). Effects of isolation rearing on startle reactivity, habituation, and prepulse inhibition in male Lewis, Sprague-Dawley, and Fischer F344 rats. Behavioral Neuroscience, 112(6), 1450-1457.

Varty, G. B., \& Higgins, G. A. (1995). Examination of drug-induced and isolation-induced disruptions of prepulse inhibition as models to screen antipsychotic drugs. Psychopharmacology, 122(1), 15-26.

Weinberger, D. R. (1987). Implications of normal brain development for the pathogenesis of schizophrenia. Archives of General Psychiatry, 44(7), 660-669.

Wilkinson, L. S., Killcross, S. S., Humby, T., Hall, F. S., Geyer, M. A., \& Robbins, T. W. (1994). Social isolation in the rat produces developmentally specific deficits in prepulse inhibition of the acoustic startle response without disrupting latent inhibition. Neuropsychopharmacology, 10(1), 61-72.

Winstanley, C. A., Theobald, D. E., Dalley, J. W., Cardinal, R. N., \& Robbins, T. W. (2006). Double dissociation between serotonergic and dopaminergic modulation of medial prefrontal and orbitofrontal cortex during a test of impulsive choice. Cerebral Cortex, 16(1), 106-114.

Woods, P. J., Fiske, A. S., \& Ruckelshaus, S. I. (1961). The effects of drives conflicting with exploration on the problem-solving behavior of rats reared in free and restricted environments. Journal of Comparative Physiology and Psychology, 54, 167-169.

Wright, C. I., Keuthen, N. J., Savage, C. R., Martis, B., Williams, D., Wedig, M., McMullin, K., \& Rauch, S. L. (2006). Brain correlates of negative and positive visuospatial priming in adults. NeuroImage, 30(3), 983-991.

Wright, C. I., McMullin, K., Martis, B., Fischer, H., \& Rauch, S. L. (2005). Brain correlates of negative visuospatial priming in healthy children. Psychiatry Research, 139(1), 41-52.

Wylie, S. A., \& Stout, J. C. (2002). Enhanced negative priming in Parkinson's disease. Neuropsychology, 16(2), 242-250.

Yamaguchi, S., \& Kobayashi, S. (1998). Contributions of the dopaminergic system to voluntary and automatic orienting of visuospatial attention. Journal of Neuroscience, 18(5), 1869-1878.

Zeeb, F. D., Wong, A. C., \& Winstanley, C. A. (2013). Differential effects of environmental enrichment, social-housing, and isolation-rearing on a rat gambling task: Dissociations between impulsive action and risky decision-making. Psychopharmacology, 225(2), 381-395. 